\title{
Heavy ion beam loss mechanisms at an electron-ion collider
}

\author{
Spencer R. Klein* \\ Lawrence Berkeley National Laboratory, Berkeley, California 94720, USA
}

(Received 17 September 2014; published 17 December 2014)

\begin{abstract}
There are currently several proposals to build a high-luminosity electron-ion collider, to study the spin structure of matter and measure parton densities in heavy nuclei, and to search for gluon saturation and new phenomena like the colored glass condensate. These measurements require operation with heavy nuclei. We calculate the cross sections for two important processes that will affect accelerator and detector operations: bound-free pair production and Coulomb excitation of the nuclei. Both of these reactions have large cross sections, 28-56 mb, which can lead to beam ion losses, produce beams of particles with altered charge:mass ratio, and produce a large flux of neutrons in zero degree calorimeters. The loss of beam particles limits the sustainable electron-ion luminosity to levels of several times $10^{32} / \mathrm{cm}^{2} / \mathrm{s}$.
\end{abstract}

DOI: 10.1103/PhysRevSTAB.17.121003

PACS numbers: 29.20.db, 25.70.De

Electron-ion colliders have been proposed as a means to study the structure functions of polarized protons and to probe the quark and gluon distributions of heavy nuclei $[1,2]$. The latter topic is of great interest in studying the behavior of quarks and gluons at high densities, such as are present in nuclei at low Bjorken- $x$ values. These studies require high electron-ion luminosities, so as to be able to probe reactions with low cross sections, near the kinematic limit in $x$ and $Q^{2}$.

At very high luminosities, other reactions, with large cross sections, may occur copiously enough to cause significant beam loss. Two such reactions are Coulomb excitation of heavy nuclei and bound-free pair production (BFPP). In BFPP, an electron-positron pair is produced, with the electron bound to the target nucleus. BFPP leads to a single-electron ion, while Coulomb excitation leads to neutron emission and/or nuclear breakup. Both reactions proceed via photon exchange between the two nuclei.

Most Coulomb excitation occurs at low photon energies. A nucleus is excited, typically to a giant dipole resonance (GDR); the GDR usually decays via single neutron emission, leaving a slightly lighter ion, plus a neutron [3]. At higher energies, Coulomb excitation can involve photon-nucleon interactions, such as excitation to a $\Delta$ resonance, or more energetic photon-quark interactions which lead to nuclear breakup and/or multiple neutron emission.

Both GDR excitation and BFPP generate a beam of ions with slightly different charge $(Z)$ to mass $(A)$ ratio, than the circulating ion beam, but with practically unchanged pernucleon momentum. These beams gradually diverge from

\footnotetext{
*srklein@lbl.gov

Published by the American Physical Society under the terms of the Creative Commons Attribution 3.0 License. Further distribution of this work must maintain attribution to the author $(s)$ and the published article's title, journal citation, and DOI.
}

the orbit of the uninteracted ions and are lost from the beam, reducing the luminosity. Depending on the beam optics, these beams may remain collimated long enough to strike the accelerator beam pipe at a specific location where they might deposit enough energy to quench superconducting magnets or generate radiation damage. This process is the main factor limiting the LHC luminosity with heavy ion beams [4-6].

In this work, we calculate the cross section for Coulomb excitation and BFPP for different proposed machine configurations. We then consider some of the consequences for the proposed designs.

Three different designs are under consideration: two approaches for a U.S.-based, moderate-energy, highluminosity electron-ion collider (EIC), and the much higher energy CERN LHeC. The Brookhaven eRHIC and the LHeC designs add electron accelerators to existing hadron colliders, while the Jefferson Laboratory MEIC builds on their existing electron accelerator. Proposed parameters for the EIC designs are listed in Ref. [1], while the LHeC is discussed in Ref. [2]. For $e A$ collisions, the EIC luminosities are presented in terms of electron-nucleon luminosities (i.e., the number of electron-nucleon collisions), rather than the conventional electron-ion luminosities, which are a factor of $A \approx 200$ lower [7].

Some key accelerator parameters are listed in Table I. Recent users group meeting presentations [8] and a new design study [9] have quoted considerably lower luminosities for the eRHIC designs, along with different beam energies, while recent MEIC presentations [10] quote $e A$ luminosities up to about 2.5 times higher. Here, we use the Ref. [1] values as baselines. At both machines, higher luminosities are also under discussion in a staged approach, either at a high-luminosity interaction region [1] or by machine upgrades. Eventual electron-nucleon luminosities up to $10^{35} / \mathrm{cm}^{2} / \mathrm{s}$ (electron-nucleus luminosity $5 \times 10^{32} / \mathrm{cm}^{2} / \mathrm{s}$ ) are envisioned $[9,11]$. 
TABLE I. Parameters for different proposed electron-ion colliders with heavy ion beams. eRHIC will use gold beams, while the MEIC and $\mathrm{LHeC}$ will accelerate lead beams. The ion energy is per nucleon, while the luminosity is the $e A$ luminosity, rather than the $e$-nucleon luminosity. For MEIC, the value for a high-luminosity interaction region is used [1].

\begin{tabular}{|c|c|c|c|c|}
\hline Accelerator & Ion energy $(\mathrm{GeV})$ & Electron energy $(\mathrm{GeV})$ & Luminosity peak $\left(\mathrm{cm}^{-2} \mathrm{~s}^{-1}\right)$ & Time between collisions \\
\hline eRHIC & 100 & 10 & $8.1 \times 10^{31}$ & $105 \mathrm{~ns}$ \\
\hline MEIC & 40 & 5 & $5.3 \times 10^{31}$ & $1.25 \mathrm{~ns}$ \\
\hline $\mathrm{LHeC}$ & 2940 & 60 & $2.2 \times 10^{29}$ & $25 \mathrm{~ns}$ \\
\hline
\end{tabular}

There are several possible LHeC configurations, involving ring-ring and continuous or pulsed ring-linac colliders [2]. Most of the designs have an electron energy of $60 \mathrm{GeV}$, but a pulsed ring-linac design avoids synchrotron radiation losses, so can reach higher electron energies-140 GeV. However, it has a lower luminosity than the other configurations. Here, we consider a continuous ring-linac configuration, with the "ultimate" per-nucleon $e A$ luminosity from Eq. (6.17) of [2], converted to per nucleus. The ringring design has the same luminosity.

As we will see, the cross sections do not depend significantly on the collision energy, but the luminosity is critical in determining the overall reaction rate.

We calculate the cross sections by using the equivalent photon approximation [12]:

$$
\sigma=\int \frac{d N}{d k} \sigma_{\gamma}(k) d k
$$

where $k$ is the photon energy in the target nucleus rest frame, $d N / d k$ is the equivalent photon spectrum, and $\sigma_{\gamma}(k)$ is the cross section for Coulomb excitation or BFPP. This approach neglects the dependence of $\sigma_{\gamma}(k)$ on the photon virtuality $\left(q^{2}\right)$. Usually, this is not a large correction. The integral runs from threshold up to the maximum allowed photon energy. Since the photon spectrum scales as $1 / k$, high photon energies are not important, and we will use a maximum energy of $k / 10$.

The photon flux from an electron with energy $E$ is

$$
\frac{d N}{d k}=\frac{\alpha}{\pi k}\left(1-\frac{k}{E}+\frac{k^{2}}{2 E^{2}}\right) \ln \left(\frac{q_{\max }^{2}}{q_{\min }^{2}}\right),
$$

where $q_{\max }^{2}$ and $q_{\min }^{2}$ span the $q^{2}$ range for the photon and $\alpha=1 / 137.04$ is the fine structure constant. For an electron with energy $E$ emitting a photon with energy $k$, the kinematic minimum is $q_{\min }^{2}=m_{e}^{2} k^{2} / E(E-k)$ [13], where $m_{e}$ is the electron mass.

For BFPP, the main contribution is at $q^{2} \approx k^{2}$ [14]; following Eq. (6.13a) of [13], we use $q_{\max }^{2}=k^{2}$, so the logarithm in Eq. (2) is $\ln \left[E(E-k) / m_{e}^{2}\right]$.

Coulomb excitation can proceed via many different subprocesses. GDR is the dominant process; it occurs for photon energies from 7-8 MeV (depending on the nucleus) up to about $24 \mathrm{MeV}$. At slightly higher energies, additional nuclear excitation channels open up. These largely lead to multiple neutron emission. At still higher energies, individual nucleons may be excited, such as to a $\Delta$ resonance. This work uses the cross sections from Baltz et al. [3,15-17]. Because of the low photon energies and large cross sections, GDR is the most important nuclear excitation process. The GDR resonance is approximated as a Lorentzian, by using the parameters in Table 3 of Ref. [18]. The cross section is taken to be zero below the measured $1 n$ threshold of $8.1 \mathrm{MeV}(7.4 \mathrm{MeV})$ for gold (lead). For the gold data (only) in Ref. [18], a Lorentzian does not appear to be a good fit. However, newer data have also been fit to a Lorentzian, albeit, for gold, with a slightly smaller $(7 \%)$ cross section but a wider GDR resonance [19]. The two sets of parameters lead to very similar overall $e A$ cross sections.

For Coulomb excitation, the $q^{2}$ range depends on the specific subreaction. GDR excitation is a collective nuclear effect with a natural maximum $q_{\max }^{2}=\left(\hbar / R_{A}\right)^{2}$, where $R_{A} \approx 7 \mathrm{fm}$ is the nuclear radius. Since this is a looser requirement than $q_{\max }^{2}=k^{2}$, we use the latter expression.

The cross section for BFPP can be derived by applying crossing symmetry to a calculation by Sauter [20] of the $K$-shell photoelectric effect [21,22]. The Sauter cross section scales as $Z^{5}$ but does not include Coulomb corrections, so is inaccurate for heavy nuclei. Multiplicative nuclear correction factors work well at high photon energies [21] but cannot reproduce the change in the shape of the cross section near threshold. We use numerical data from an exact calculation, the $Z=92$ curve in Fig. 1 of Ref. [22], scaled by $Z^{5}$ for energies below $14.5 \mathrm{MeV}$. At higher energies, we use the Sauter formula with the high- $Z$ correction from Eqs. (9) and (10) of [22]:

$$
\sigma=\frac{4 \pi \lambda_{c}^{2} Z^{5} f(Z)}{k}
$$

where

$$
f(Z)=\left(\frac{53}{100}+\frac{2 \pi \alpha Z}{9}\right)^{2 \pi \alpha Z}
$$

and $\lambda_{c}=386 \mathrm{fm}$ is the electron Compton wavelength. These should agree to within $5 \%$ of the exact cross sections [23]. We increase the BFPP cross sections by $20 \%$, to account for electron capture to higher orbitals [21]. 
For the $\mathrm{LHeC}$, there is an additional complication. Equation (2) gives the total photon flux, integrated out to infinite electron-ion impact parameters. For a given photon energy, the maximum impact parameter is $b_{\max }=\Gamma \hbar c / k$, where $\Gamma$ is the Lorentz boost of the electron in the ion rest frame. For a $1 \mathrm{MeV}$ photon, $b_{\max }=$ 136(317) $\mu \mathrm{m}$ for the continuous (pulsed) version, larger than the radius of the colliding beams (the heavy ion ring retains the LHC optics, so $R=15.9 \mu \mathrm{m}$ [24]; for LHeC designs with a nonzero beam crossing angle, the effective size may be larger). The photon flux at larger impact parameters should not be included in the total. Avoiding the extra flux requires an impact-parameter-sensitive formulation of the photon flux and some knowledge of the distribution of particles in the beam. The beam size limitation applies for $k<k_{c}=8.7 \mathrm{MeV}$, so it affects BFPP but not Coulomb excitation. For $140 \mathrm{GeV}$ electron beams at the $\mathrm{LHeC}, k_{c}=20 \mathrm{MeV}$, so the beam size also affects Coulomb excitation, but the overall reduction in cross section is slightly less than $1 \%$.

One simple way to estimate the magnitude of the reduction is to set $q_{\min }=\hbar / R$ - the transverse momentum uncertainty due to the localization-when $k<k_{c}$. This produces a smooth transition at $k=k_{c}$. For a $2.5 \mathrm{MeV}$ photon at $\mathrm{LHeC}-1$, the finite beam radius reduces the photon flux by about $6 \%$. The overall reduction in cross section is also about $6 \%$. A more detailed calculation is beyond the scope of this paper, but the magnitude of the reduction provides an estimate of the associated uncertainty. This beam-size effect has been observed at the VEPP-4 $e^{+} e^{-}$collider and the HERA $e p$ collider [25].

The main uncertainty in the cross sections is neglect of the photon $q^{2}$ in $\sigma_{\gamma}(k)$. One study found an ambiguity of up to a factor of 2 in the Weizsäcker-Williams approach due to uncertainties in the $q^{2}$ cutoffs [14]. However, uncertainties in the $q^{2}$ range enter only logarithmically in the total cross section. At an electron-ion collider, the large Lorentz boosts lead to a very small $q_{\min }$; very small $q^{2}$ will not have a large effect on $\sigma_{\gamma}(k)$. Hundley [26] found good agreement between the Weizsäcker-Williams method and a quantum electrodynamics calculation of direct (unbound) pair production. Overall, the cross sections should be accurate to $25 \%$.

The top panel of Fig. 1 shows the Coulomb excitation and BFPP cross sections. The BFPP curve peaks around $1.4 \mathrm{MeV}$ and decreases slowly at higher energies. The threshold is less than $2 m_{e}$ because of the electron binding energy. The slight discontinuity at $14.5 \mathrm{MeV}$ signals the switch from the exact calculation to the Sauter formula. The Coulomb excitation curve is dominated by the giant dipole resonance. The second peak, around $300 \mathrm{MeV}$, is from nucleon excitation to a $\Delta$ resonance. The bottom panel of the figure shows the cross sections weighted by the photon flux $(\sigma \times d N / d k$, essentially $\sigma / k)$, showing that lowenergy photons dominate the cross sections.
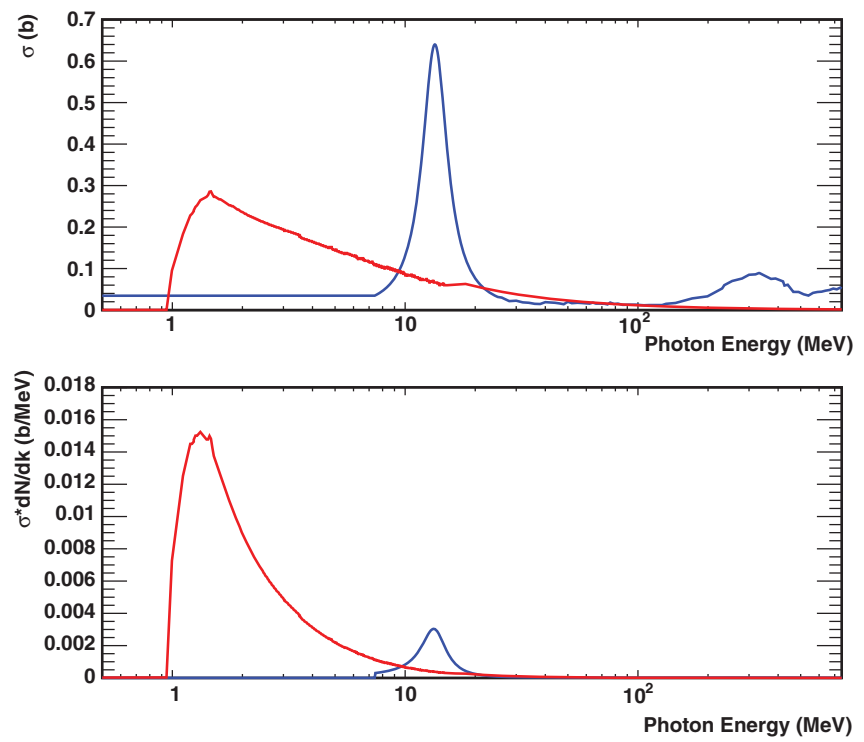

FIG. 1. (Top) Per-photon cross section for bound-free (blue line) and Coulomb excitation (red line) for gold beams at eRHIC, as a function of photon energy, in the target rest frame. (Bottom) Flux-weighted cross sections for $e A$ production of Coulomb excited gold and BFPP. The photon spectrum scales as $1 / k$, so the reactions are concentrated at low photon energies.

The 2nd and 3rd rows of Table II show the cross sections for BFPP and Coulex. The BFPP cross sections are about $20 \%$ larger than those for Coulex. Both show only slow variation with collision energy, because the energy dependence enters mainly through the logarithmic term in Eq. (2). For BFPP, the LHeC cross sections are almost identical for 60 and $140 \mathrm{GeV}$ electrons, because, at the few-MeV photon energies that dominate the cross section, the photon flux is constrained by the beam radius, leading to identical logarithmic factors in Eq. (2).

These cross sections are reasonably close to the cross sections found for the corresponding ultraperipheral heavy ion interactions, after scaling downward by the square of the ion charge, to account for the reduced photon flux. For example, at the LHC, the cross section for BFPP in leadlead collisions is $281 \mathrm{~b}$, and that for Coulomb excitation is $220 \mathrm{~b}$ [15]. Scaling downward by $1 / 82^{2}$ leads to cross sections of 41 and $34 \mathrm{mb}$, similar to the 56 and $45 \mathrm{mb}$ for the corresponding $e A$ processes. The $e A$ cross sections are

TABLE II. Cross sections and reaction rates for BFPP and Coulex and the beam power for BFPP and GDR excitation.

\begin{tabular}{lrrr}
\hline \hline Parameter & eRHIC & MEIC & LHeC \\
\hline$\sigma$ (BFPP) & $37 \mathrm{mb}$ & $39 \mathrm{mb}$ & $56 \mathrm{mb}$ \\
$\sigma$ (Coulex) & $31 \mathrm{mb}$ & $28 \mathrm{mb}$ & $45 \mathrm{mb}$ \\
BFPP particle/s & $3.0 \times 10^{6}$ & $2.1 \times 10^{6}$ & $1.2 \times 10^{4}$ \\
Coulex particle/s & $2.5 \times 10^{6}$ & $1.5 \times 10^{6}$ & $1.0 \times 10^{4}$ \\
BFPP beam power & $9.3 \mathrm{~W}$ & $2.8 \mathrm{~W}$ & $1.1 \mathrm{~W}$ \\
GDR beam power & $5.3 \mathrm{~W}$ & $1.3 \mathrm{~W}$ & $0.6 \mathrm{~W}$ \\
\hline \hline
\end{tabular}


of the order of $50 \%$ larger than the scaled estimates, primarily because of the larger electron Lorentz boost, resulting in a large logarithmic factor in Eq. (2). The $e A$ cross sections show less energy dependence going from RHIC or EIC energies to $\mathrm{LHC}$ or $\mathrm{LHeC}$ energies than is seen for ion-ion collisions. And, at LHC energies, the BFPP cross section is almost independent of the collision energy, in contrast to the ion-ion case.

The 4th and 5th rows of the table show the reaction rates: the Table I luminosities multiplied by the cross sections. The 6th and 7th rows show the power carried by the beam of altered nuclei. These beam losses can have consequences for machine and detector operations. With both processes, the target ion is lost from the beam, decreasing the luminosity. The neutrons produced from Coulex are also a significant background for experiments, particularly for studies of neutron-free interactions like coherent photoproduction.

Since the momentum transfer to the ion system is a very small fraction of the total ion momentum, both Coulex and BFPP create a collimated beam of ions with an altered $Z / A$. These beams deposit energy wherever they hit the beam pipe. The GDR cross section is about $2 / 3$ of the total Coulomb excitation cross section [15]. At RHIC, these ions will lose their collimation (i.e., spread out) before striking the beam pipe and so will distribute their energy around the accelerator ring [27]. At the $\mathrm{LHeC}$, they will strike the beam pipe in a well-defined location. Although the power levels are low for the parameters in Table I, they could be significant at a higher luminosity collider.

Both Coulomb excitation and BFPP remove ions from the beam and so reduce the luminosity. In 2014, RHIC ran gold-gold collisions at a center of mass energy of $200 \mathrm{GeV} /$ nucleon, with 111 bunches, each containing $1.6 \times 10^{9}$ particles, for a total of $1.78 \times 10^{11}$ circulating ions [28]. Assuming that eRHIC has similar parameters, a loss rate of $5.5 \times 10^{6}$ ions $/ \mathrm{s}$ (at $8.1 \times 10^{31} / \mathrm{cm}^{2} / \mathrm{s} \mathrm{lumi-}$ nosity) from BFPP plus Coulomb excitation leads to a beam lifetime of $9 \mathrm{~h}$ for one interaction region or $41 / 2 \mathrm{~h}$ with two interaction points. The September 2014 eRHIC Design Study, however, anticipated lower beam intensities, $0.6 \times 10^{9}$ particles/bunch [9]. At these intensities and the Table I eRHIC luminosity, the beam lifetimes would be shorter, 200 mins with 1 IR or 100 min with two IRs. At the luminosity presented in the design study, the beam lifetime is long enough to avoid trouble.

Coulomb excitation also produces one or more neutrons, which leave signals in zero degree calorimeters. The eRHIC neutron production rate of 2.5 Mevents/s should be compared to the beam crossing rate of $9.5 \mathrm{MHz}$. Each crossing will contain an average of $1 / 4$ interactions, each with one or more neutrons. These neutrons are a background contamination for other reactions, particularly for coherent photonuclear interactions where the nucleus remains intact, since the signature for nuclear survival is the absence of neutrons. The MEIC has a 1.25 nsec interval between collisions, so the neutron background may be largely removed via the use of a calorimeter with good timing.

These issues become problematic at the higher luminosities. At a luminosity of $5 \times 10^{32} / \mathrm{cm}^{2} / \mathrm{s}$ with $0.6 \times 10^{9}$ particles/bunch, the eRHIC beam lifetime would be a prohibitively short 34 (17) min with 1 (2) IRs. There would be $11 / 2$ neutron-producing interactions per beam crossing, greatly reducing the efficiency for selecting neutron-free coherent events. Depending on the machine design, the $50 \mathrm{~W}$ of beam power carried by the beam of single-electron ions could be problematic. It should be noted that fairly high luminosities are required to accomplish the $e A$ physics goals articulated in Ref. [1] in a timely manner; most of the $e A$ plans are based on $10 \mathrm{fb}^{-1} / A$ of integrated (per nucleon) luminosity.

In conclusion, we have considered two processes which will have large cross sections at proposed electron-ion colliders: bound-free pair production and Coulomb excitation. Both processes have small effects on the current accelerator designs but would present significant obstacles for higher-luminosity accelerators.

We thank John Jowett and Tom Roser for help understanding $e A$ luminosities and Peter Jacobs for editorial suggestions. This material is based upon work supported in part by the U.S. Department of Energy, Office of Science, Office of Nuclear Physics, under Contract No. DE-AC02$05 \mathrm{CH} 11231$.

[1] A. Accardi et al., arXiv:1212.1701.

[2] J. L. Abelleira Fernandez et al. (LHeC Study Group Collaboration), J. Phys. G 39, 075001 (2012).

[3] A. J. Baltz, M. J. Rhoades-Brown, and J. Weneser, Phys. Rev. E 54, 4233 (1996).

[4] S. R. Klein, Nucl. Instrum. Methods Phys. Res., Sect. A 459, 51 (2001).

[5] R. Bruce, S. Gilardoni, J. M. Jowett, and D. Bocian, Phys. Rev. ST Accel. Beams 12, 071002 (2009).

[6] J. Jowett, Workshop on Photon Induced Collisions at the LHC, 2014, CERN, Geneva (unpublished). Slides available at https://indico.cern.ch/event/216417/contribution/ 2/material/slides/.

[7] T. Roser (personal communication).

[8] V. Litvenko, Electron Ion Collider Users Group Meeting, 2014, Stony Brook University, New York (unpublished). Slides available at http://skipper.physics.sunysb.edu/ abhay/ 2014/EICUM/Talks/06252014-AM/2_Litvinenko.pptx.

[9] E. C. Aschenauer et al., arXiv:1409.1633.

[10] F. Pilat, Electron Ion Collider Users Group Meeting, 2014, Stony Brook University, New York (unpublished). Slides available at http://skipper.physics.sunysb.edu/ abhay/ 2014/EICUM/Talks/06252014-AM/1_Pilat.ppt.

[11] See, for example, https://wiki.bnl.gov/eic/index.php/ Luminosity. 
[12] C. A. Bertulani, S. R. Klein, and J. Nystrand, Annu. Rev. Nucl. Part. Sci. 55, 271 (2005).

[13] V. M. Budnev, I. F. Ginzburg, G. V. Meledin, and V. G. Serbo, Phys. Rep. 15, 181 (1975).

[14] C. A. Bertulani and G. Baur, Phys. Rev. D 58, 034005 (1998).

[15] A. J. Baltz, C. Chasman, and S. N. White, Nucl. Instrum. Methods Phys. Res., Sect. A 417, 1 (1998).

[16] A. J. Baltz, S. R. Klein, and J. Nystrand, Phys. Rev. Lett. 89, 012301 (2002).

[17] A. J. Baltz, Y. Gorbunov, S. R. Klein, and J. Nystrand, Phys. Rev. C 80, 044902 (2009).

[18] A. Veyssiere, H. Beil, R. Bergere, P. Carlos, and A. Lepretre, Nucl. Phys. A159, 561 (1970).

[19] B. L. Berman, R. E. Pywell, S.S. Dietrich, M. N. Thompson, K. G. McNeill, and J. W. Jury, Phys. Rev. C 36, 1286 (1987).
[20] F. Sauter, Ann. Phys. (Berlin) 403, 454 (1931).

[21] C. K. Agger and A. H. Sorensen, Phys. Rev. A 55, 402 (1997).

[22] A. W. Aste, Europhys. Lett. 81, 61001 (2008).

[23] Figure 12 of A.H. Sørensen, Phys. Rev. A 64, 012703 (2001) shows that the Sauter expression should be accurate to within $10 \%$.

[24] J. Beringer et al. (Particle Data Group Collaboration), Phys. Rev. D 86, 010001 (2012), Sec. 29.

[25] G. L. Kotkin and V. G. Serbo, Phys. Rev. ST Accel. Beams 7, 101001 (2004).

[26] R. O. Hundley, Ph.D. dissertation, Caltech, 1963.

[27] R. Bruce, J. Jowett, S. Gilardoni, A. Drees, W. Fischer, S. Tepikian, and S. Klein, Phys. Rev. Lett. 99, 144801 (2007).

[28] http://www.agsrhichome.bnl.gov/RHIC/Runs/. 